\title{
Constraints and Adaptation of Closed-Loop Neuroprosthetics for Functional Restoration
}

\section{OPEN ACCESS}

Edited by:

Mikhail Lebedev,

Duke University, USA

Reviewed by:

ShiNung Ching,

Washington University in St. Louis,

USA

Aysegul Gunduz,

University of Florida, USA

${ }^{*}$ Correspondence:

Robert Bauer

robert.bauer@cin.uni-tuebingen.de

Alireza Gharabaghi

alireza.gharabaghi@uni-tuebingen.de

Specialty section: This article was submitted to Neuroprosthetics,

a section of the journal

Frontiers in Neuroscience

Received: 05 June 2016 Accepted: 21 February 2017

Published: 13 March 2017

Citation:

Bauer R and Gharabaghi A (2017)

Constraints and Adaptation of

Closed-Loop Neuroprosthetics for

Functional Restoration.

Front. Neurosci. 11:111

doi: 10.3389/fnins.2017.00111

\author{
Robert Bauer * and Alireza Gharabaghi * \\ Division of Functional and Restorative Neurosurgery, Centre for Integrative Neuroscience, Eberhard Karls University \\ Tuebingen, Tuebingen, Germany
}

Closed-loop neuroprosthetics aim to compensate for lost function, e.g., by controlling external devices such as prostheses or wheelchairs. Such assistive approaches seek to maximize speed and classification accuracy for high-dimensional control. More recent approaches use similar technology, but aim to restore lost motor function in the long term. To achieve this goal, restorative neuroprosthetics attempt to facilitate motor re-learning and to strengthen damaged and/or alternative neural connections on the basis of neurofeedback training within rehabilitative environments. Such a restorative approach requires reinforcement learning of self-modulated brain activity which is considered to be beneficial for functional rehabilitation, e.g., improvement of $\beta$-power modulation over sensorimotor areas for post-stroke movement restoration. Patients with motor impairments, however, may also have a compromised ability for motor task-related regulation of the targeted brain activity. This would affect the estimation of feature weights and hence the classification accuracy of the feedback device. This, in turn, can frustrate the patients and compromise their motor learning. Furthermore, the feedback training may even become erroneous when unconstrained classifier adaptation-which is often used in assistive approaches - is also applied in this rehabilitation context. In conclusion, the conceptual switch from assistance toward restoration necessitates a methodological paradigm shift from classification accuracy toward instructional efficiency. Furthermore, a constrained feature space, a priori regularized feature weights, and difficulty adaptation present key elements of restorative brain interfaces. These factors need, therefore, to be addressed within a therapeutic framework to facilitate reinforcement learning of brain self-regulation for restorative purposes.

Keywords: assistive technology, neurorehabilitation, stroke, rehabilitation robotics, brain-computer interface, brain-machine interface, brain-robot interface

\section{RESTORATION INSTEAD OF ASSISTANCE}

Brain self-regulation has recently been applied in the context of motor rehabilitation after stroke by providing contingent feedback of motor imagery (Buch et al., 2012; Ang et al., 2014; Morone et al., 2015; Pichiorri et al., 2015). In these approaches, specific brain states (i.e., rest vs. motor imagery) are often separated using an online analysis of sensorimotor power in a cue-paced trial-structure. When used in conjunction with robotic rehabilitation technology, these devices are also referred to as brain-robot interfaces (BRI; Bauer et al., 2015; Naros and Gharabaghi, 2015; Kraus et al., 2016). 
While assistive BRIs aim to replace lost function by controlling external devices (Hochberg et al., 2012; Collinger et al., 2013), restorative BRIs aim to rehabilitate an impaired function (Gharabaghi, 2016; Krucoff et al., 2016). In such a restorative framework, BRIs adhere to an operant conditioning rationale (Sherlin et al., 2011; Bauer and Gharabaghi, 2015b). They provide contingent feedback to facilitate the self-regulation of specific brain activity. This reinforcement learning-based approach is considered to be beneficial for recovery and might ultimately lead to functional gains on the basis of motor re-learning and strengthening of damaged and/or alternative neural connections (Daly and Wolpaw, 2008). Restorative BRIs might be additionally supported by brain state dependent stimulation to strengthen cortico-spinal connectivity (Gharabaghi et al., 2014a; Royter and Gharabaghi, 2016; Kraus et al., 2016).

\section{METHODOLOGICAL ADJUSTMENTS}

We propose that, on account of their different goals, these restorative techniques require a different methodological approach than assistive BRIs, i.e., modifying brain physiology vs. controlling extremal devices. We acknowledge that different strategies may be adopted to achieve modified neurophysiology and, ultimately, behavioral gains. However, on the basis of empirical evidence acquired in our lab, we propose the following adjustments: constrained feature space, regularized feature weights, and difficulty adaptation.

Instead of analyzing all acquired signals for optimal classification, we propose that the feature space be intentionally constrained to reinforce a specific oscillatory pattern in accordance with the respective treatment rationale (constrained feature space). In a next step, to differentiate between the classes, assistive BRIs use classifier calibration to weight features according to their relevance. However, learning brain selfregulation may lead to non-stationarity of these classes in the course of the training (Vidaurre et al., 2011a; Sugiyama et al., 2013; Naros and Gharabaghi, 2015). Unsupervised adaptation of the feature weights may therefore lead to a switch in the mental strategy (Vidaurre et al., 2011b; Bryan et al., 2013). This approach may even result in artefactual control (Gharabaghi et al., 2014b). $\mathrm{We}$, therefore, propose that feature weight regularization be applied to address this issue. Furthermore, cognitive, sensory, and motor impairments may limit the ability to modulate brain activity, perceive, and/or process feedback. This may cause frustration, which, in turn, may be exacerbated due to the low classification accuracy caused by the constrained and regularized feature space (Nijboer et al., 2008; Fels et al., 2015). In this context, we propose that difficulty adaptation be applied to overcome cognitive load issues (Bauer and Gharabaghi, 2015a; Bauer et al., 2016a,b). Such an approach may also improve the instructional efficiency of feedback (Bauer and Gharabaghi, 2015b) and maintain motivation (Bauer et al., 2016a,b).

In the following paragraphs, we discuss these methodological adjustments in greater detail.

\section{CONSTRAINED FEATURE SPACE}

In high-dimensional feature spaces, some regions may be sparsely populated with data, thereby, impairing the classifier setup (Theodoridis and Koutroumbas, 2009). Under these circumstances, constraining the feature space provides a way of dealing with this curse of dimensionality. However, if the feature space is constrained a priori, some useful features for classification may also be discarded. A classifier based on a constrained feature set therefore usually performs less well than a classifier based on a full feature set.

Restorative BRIs, which apply this approach, therefore appear inferior in comparison to their assistive counterparts. The latter use more flexible algorithms to select and weight all available features and to maximize classification accuracy (Ang et al., 2009; Theodoridis and Koutroumbas, 2009). An a priori constraint should therefore be well considered. It is tempting to assume that the brain will find the best combination of features by itself. Such an approach is therefore implicitly followed during standard or robotic neurorehabilitation, when the feedback that is provided by the therapist or the training device is independent of specific brain features. This strategy, however, has not been successful until now, at least when considering severely motor-impaired stroke patients with persistent deficits. Moreover, the features ( $\alpha$-desynchronization) identified as most useful for classification between different states in the post-stroke brain, e.g., rest vs. motor imagery, are not necessarily those that are most therapeutically relevant ( $\beta$-desynchronization): Synchronization/Desynchronization describe the (often taskinduced) increase/reduction in power in specific frequency bands. The $\alpha$-band usually ranges from $8-14 \mathrm{~Hz}$, while the $\beta$-band ranges from $15-30 \mathrm{~Hz}$. Specifically, movementrelated $\beta$-desynchronization ( $\beta$-ERD) is compromised in the contralateral primary cortex in comparison to healthy controls; the more severe the patient's motor impairment, the less $\beta$ ERD (Rossiter et al., 2014). And so $\beta$-ERD remains inferior to other features for classification purposes in stroke patients, e.g., in differentiating movement-related brain states for the control of external devices (Gomez-Rodriguez et al., 2011). In this context, we argue that the fact that $\beta$-oscillations are less optimal for classification purposes does not compromise-but rather qualifies-this physiological marker as a therapeutic target (Naros and Gharabaghi, 2015). Here, we see an analogy to the concept of constraint-induced movement therapy in stroke patients, where the affected rather than the healthy side of the body is trained to facilitate restoration instead of compensation of motor function. Notably, such an approach does not exclude the possibility that alternative cortico-spinal pathways which do not originate from the contralateral primary motor cortex take over lost function. These pathways would be facilitated on the basis of cortical disinhibition and coherent interaction with the muscles in the $\beta$-band as well (Mima et al., 2001; Kilavik et al., 2012; Aumann and Prut, 2015; Brittain et al., 2014; Rossiter et al., 2014; Kraus et al., 2016).

Furthermore, an approach based on a constrained feature space allows making a direct, hypothesis-driven comparison of different interventions based on specific oscillatory patterns. By 
way of example, an increase in the $\beta$-modulation range will improve cortico-spinal connectivity (Kraus et al., 2015, 2016) and motor function (Naros et al., 2016). This will enable us to empirically detect functionally relevant markers and mechanisms of restoration and to determine physiology-based strategies for further improvement. Such knowledge will also enable us to develop approaches for treatment matching, e.g., defining feature sets on the basis of specific functional impairments and/or lesion locations (Shelton and Reding, 2001; Stinear et al., 2012). By contrast, an approach based on an unconstrained feature space would be based on the assumption that the most accurate detection of motor intention/imagery and provision of feedback is in itself sufficient to restore function.

\section{REGULARIZED FEATURE WEIGHTS}

Regularization can be considered a penalty term to prevent feature weights from reaching implausibly high values (Theodoridis and Koutroumbas, 2009; Bishop, 2013) caused by the empirical estimation of class parameters (e.g., mean and covariance). Such estimates can be biased, especially when the sample size is low. Due to the large variety of classification approaches (Theodoridis and Koutroumbas, 2009), several regularization approaches have been suggested, e.g., pooled covariance estimation (Friedman, 1989), rejection of eigenvectors (Blankertz et al., 2008), shrinkage estimators (Beltrachini et al., 2010), or feature subset selection (Friedman, 1989).

Even when recognizing that a constrained feature space can already be considered a form of regularization, the empirical determination of feature weights during the calibration period may pose a particular challenge for restorative brain-interface approaches, e.g., when estimating mean and covariance of two classes (rest vs. motor imagery). When patients are able to desynchronize sensorimotor oscillations (Pfurtscheller et al., 2005; Neuper et al., 2006; Kaiser et al., 2011), the estimation of feature weights is usually straightforward. In such a case, several approaches for regularization have been discussed (Yuan and Bentler, 1998; Beltrachini et al., 2010). If, however, the volitional modulation of sensorimotor oscillations has not been learned (Brauchle et al., 2015; Bauer and Gharabaghi, 2015b; Naros and Gharabaghi, 2015), or when it is impaired due to the underlying pathology (Buch et al., 2012; Bundy et al., 2012; Rossiter et al., 2014), the estimation might become noisy or even false. More formally, if one class (i.e. motor imagery) is not sufficiently expressed, its parameters (e.g., mean and covariance) cannot be measured. If, however, the mean during motor imagery is not sufficiently different from the mean during rest, a noisy estimate can result in the classifier being calibrated toward the wrong direction of modulation. Subsequently, the patient might receive feedback for synchronizing instead of desynchronizing.

Novelty detection has been suggested as a solution, if no information about a second class is available (Pimentel et al., 2014). Such a one-class approach might base mean and covariance estimation on the rest class only. However, without a priori information about the targeted direction of modulation, data-driven regularization approaches cannot be sufficient.

Furthermore, when a patient alters the mental strategy in the course of the intervention, a classifier trained on the initial strategy can become misaligned. In classical brain-interface approaches, the adaptation of feature weights has been proposed for such cases (Vidaurre et al., 2011b; Bryan et al., 2013; Sugiyama et al., 2013). But such data-driven approaches can be problematic for restorative approaches; classifier adaptation might condition the patients to explore alternative, i.e., therapeutically nondesired strategies (Bauer and Gharabaghi, 2015a,b). When the patient becomes frustrated with motor imagery, he/she may use artifacts for control, e.g., muscle contractions (Gharabaghi et al., 2014b).

Bearing these points in mind, we suggest employing informed regularization determined by a priori selected feature weights, thereby, ensuring the targeted direction of modulation. In our lab, we currently employ a variant of novelty classification by using a linear discriminant analysis with a fixed direction. In that regard, we base the mean and covariance estimation on the rest class only, with the parameter estimation pooled across several electrodes. Thereby, we provide feedback for the reduction of the mean, i.e., desynchronization, only.

\section{DIFFICULTY OF ADAPTATION}

Lotte and colleagues have pointed out that most neurofeedback protocols are limited with regard to their instructional design. They suggested adaptive training approaches, i.e., the use of difficulty levels which are challenging, but still achievable (Lotte et al., 2013). A similar idea was postulated by the cognitive load theory (Schnotz and Kürschner, 2007). On the basis of these concepts, both under- and over-challenge must be avoided to facilitate learning (Schnotz and Kürschner, 2007; Bauer and Gharabaghi, 2015a). In classifiers, which are constrained, regularized and linear, item response theory enables us to directly relate the threshold used for classification to the difficulty level (Bauer and Gharabaghi, 2015a). By using a linear discriminant analysis with a fixed direction, thresholding allows us to provide reward for desynchronization only when it is sufficiently strong. Within this framework, the shape of classification accuracy CA across different threshold can be interpreted as the zone of proximal development (ZPD). This argument, with detailed examples, has been clarified elsewhere (Bauer and Gharabaghi, 2015a). The ZPD is an indirect measure of a subject's cognitive resources (Schnotz and Kürschner, 2007). It also constitutes the range of threshold, where learning may occur because subjects are able to compensate for the extraneous load caused by the mismatch of ability and difficulty (Bauer and Gharabaghi, 2015a). Along these lines, two recent studies with healthy subjects provided empirical evidence that dynamic threshold adaptation is instrumental in facilitating learning (Bauer et al., 2016b; Naros et al., 2016).

Unconstrained and unregularized classifiers do not offer an accessible, one-dimensional parameter to fine-tune the difficulty of the task. It might therefore be problematic to adapt 
the difficulty within these approaches. In particular, a multidimensional or even non-linear theory of difficulty adaptation appears to be challenging. We instead explored the difficulty threshold of a linear, a priori constrained and regularized classifier and found evidence of a direct correlation between the subjects' perceived mental effort and the task difficulty (Bauer et al., 2016b). Further empirical evidence suggests that there is a link between classification accuracy and cognitive load; classification performance has been linked to mood and mastery confidence (Nijboer et al., 2008), as well as to the degree of concentration on the task and the ability to ignore distracting stimuli (Hammer et al., 2012). The sensation of challenge might therefore be linked to the ratio of true to false positives returned by the classifier. This hypothesis is supported by a Bayesian simulation study of reinforcement learning under adaptive changes of true and false positive rates (Bauer and Gharabaghi, 2015b). A generalized concept of difficulty adaptation might, therefore, be based on controlling the relationship between true and false positive rates by asking the patients to self-rate the perceived effort and/or applying non-cued training.

Nonetheless, further factors may affect the difficulty of the training: the challenge of achieving a sense of cognitive and internal control (Burde and Blankertz, 2006; Wood et al., 2014), the appropriate processing of cues to reduce impairments in mental chronometry (Liepert et al., 2012), and to increase the quality of motor imagery (Heremans et al., 2009, 2012), the specific sensory impairments of patients and their interaction with the feedback modality (e.g., visual, haptic, auditory) (Nijboer et al., 2008; Gomez-Rodriguez et al., 2011; Parker et al., 2011; Sollfrank et al., 2015), or the repetitive and fatiguing nature of training (Lee et al., 1991; Page et al., 2011). Dealing with these aspects by proper instructional design is more important for restorative than for assistive approaches (Lotte et al., 2013).

\section{CONCLUSION}

We propose that restorative approaches should apply prior information about beneficial features (e.g., $\beta$-power desynchronization over sensorimotor areas) to constrain the

\section{REFERENCES}

Ang, K. K., Chin, Z. Y., Zhang, H., and Guan, C. (2009). "Robust filter bank common spatial pattern (RFBCSP) in motor-imagery-based brain-computer interface," in Conference Proceedings Annual International Conference of the IEEE Engineering in Medicine and Biology Society (Minneapolis, MN), 578-581. doi: 10.1109/IEMBS.2009.5332817

Ang, K. K., Guan, C., Phua, K. S., Wang, C., Zhou, L., Tang, K. Y., et al. (2014). Brain-computer interface-based robotic end effector system for wrist and hand rehabilitation: results of a three-armed randomized controlled trial for chronic stroke. Front. Neuroeng. 7:30. doi: 10.3389/fneng.2014.00030

Aumann, T. D., and Prut, Y. (2015). Do sensorimotor $\beta$-oscillations maintain muscle synergy representations in primary motor cortex? Trends Neurosci. 38, 77-85. doi: 10.1016/j.tins.2014.12.002

Bauer, R., Fels, M., Royter, V., Raco, V., and Gharabaghi, A. (2016a). Closed-loop adaptation of neurofeedback based on mental effort facilitates feature space and regularize their direction. Such an approach may reduce the classification accuracy in comparison to unconstrained or unregularized approaches, particularly in patients who are only partially able to self-regulate the targeted brain state. At the same time, this method would increase the likelihood that feedback is provided for the therapeutically targeted modulation of brain activity only. The threshold selection in restorative approaches should therefore not be misled by the goal of maximum classification accuracy. Instead, it should follow instructional demands to maximize learning.

Accordingly, several methods have been proposed for locating the threshold for maximum learning (Ivanova et al., 2005; Cegarra and Chevalier, 2008; Naros et al., 2016; Bauer et al., 2016a,b). Moreover, physiological parameters, e.g., distributed cortical patterns in the $\alpha$-range (Vukelić et al., 2014; Vukelić and Gharabaghi, 2015a,b) and the $\theta$-range (Fels et al., 2015) which were linked to $\beta$-band self-regulation may also be used in the long term for this purpose.

The conceptual switch from assistive to restorative neuroprosthetics necessitates methodological adjustments (constrained feature space, a priori regularized feature weights, difficulty adaptation) which ultimately represent a paradigm switch from classification accuracy toward instructional efficiency to facilitate reinforcement learning of brain self-regulation.

\section{AUTHOR CONTRIBUTIONS}

All authors listed, have made substantial, direct and intellectual contribution to the work, and approved it for publication.

\section{ACKNOWLEDGMENTS}

RB was supported by the Graduate Training Centre of Neuroscience, International Max Planck Research School for Cognitive and Systems Neuroscience, Tuebingen, Germany. AG was supported by grants from the German Federal Ministry of Education and Research [BMBF IMONAS 13GW0119B] and the Baden-Württemberg Stiftung [NemoPlast NEU005]. There are no conflicts of interests. reinforcement learning of brain self-regulation. Clin. Neurophysiol. 127, 3156-3164. doi: 10.1016/j.clinph.2016.06.020

Bauer, R., Fels, M., Vukelić, M., Ziemann, U., and Gharabaghi, A. (2015). Bridging the gap between motor imagery and motor execution with a brainrobot interface. Neuroimage 108, 319-327. doi: 10.1016/j.neuroimage.2014. 12.026

Bauer, R., and Gharabaghi, A. (2015a). Estimating cognitive load during self-regulation of brain activity and neurofeedback with therapeutic brain-computer interfaces. Front. Behav. Neurosci. 9:21. doi: 10.3389/fnbeh.2015.00021

Bauer, R., and Gharabaghi, A. (2015b). Reinforcement learning for adaptive threshold control of restorative brain-computer interfaces: a Bayesian simulation. Front. Neurosci. 9:36. doi: 10.3389/fnins.2015.00036

Bauer, R., Vukelić, M., and Gharabaghi, A. (2016b). What is the optimal task difficulty for reinforcement learning of brain self-regulation? Clin. Neurophysiol. 127, 3033-3041. doi: 10.1016/j.clinph.2016.06.016 
Beltrachini, L., von Ellenrieder, N., and Muravchik, C. H. (2010). Shrinkage approach for EEG covariance matrix estimation. Conf. Proc. IEEE Eng. Med. Biol. Soc. 2010, 1654-1657. doi: 10.1109/iembs.2010.5626668

Bishop, C. M. (2013). Pattern Recognition and Machine Learning. New Delhi: Springer.

Blankertz, B., Tomioka, R., Lemm, S., Kawanabe, M., and Muller, K. (2008). Optimizing spatial filters for robust EEG single-trial analysis. IEEE Signal Process. Mag. 25, 41-56. doi: 10.1109/MSP.2008.4408441

Brauchle, D., Vukelić, M., Bauer, R., and Gharabaghi, A. (2015). Brain statedependent robotic reaching movement with a multi-joint arm exoskeleton: combining brain-machine interfacing and robotic rehabilitation. Front. Hum. Neurosci. 9:564. doi: 10.3389/fnhum.2015.00564

Brittain, J.-S., Sharott, A., and Brown, P. (2014). The highs and lows of beta activity in cortico-basal ganglia loops. Eur. J. Neurosci. 39, 1951-1959. doi: 10.1111/ejn.12574

Bryan, M. J., Martin, S. A., Cheung, W., and Rao, R. P. (2013). Probabilistic co-adaptive brain-computer interfacing. J. Neural Eng. 10:66008. doi: 10.1088/1741-2560/10/6/066008

Buch, E. R., Modir Shanechi, A., Fourkas, A. D., Weber, C., Birbaumer, N., and Cohen, L. G. (2012). Parietofrontal integrity determines neural modulation associated with grasping imagery after stroke. Brain J. Neurol. 135, 596-614. doi: 10.1093/brain/awr331

Bundy, D. T., Wronkiewicz, M., Sharma, M., Moran, D. W., Corbetta, M., and Leuthardt, E. C. (2012). Using ipsilateral motor signals in the unaffected cerebral hemisphere as a signal platform for braincomputer interfaces in hemiplegic stroke survivors. J. Neural Eng. 9:36011. doi: 10.1088/1741-2560/9/3/036011

Burde, W., and Blankertz, B. (2006). "Is the locus of control of reinforcement a predictor of brain-computer interface performance?," in Proceedings of the $3 \mathrm{rd}$ International Brain-Computer Interface Workshop and Training Course (Graz), 76-77.

Cegarra, J., and Chevalier, A. (2008). The use of Tholos software for combining measures of mental workload: toward theoretical and methodological improvements. Behav. Res. Methods 40, 988-1000. doi: 10.3758/BRM.40.4.988

Collinger, J. L., Wodlinger, B., Downey, J. E., Wang, W., Tyler-Kabara, E. C., Weber, D. J., et al. (2013). High-performance neuroprosthetic control by an individual with tetraplegia. Lancet 381, 557-564. doi: 10.1016/S0140-6736(12)61816-9

Daly, J. J., and Wolpaw, J. R. (2008). Brain-computer interfaces in neurological rehabilitation. Lancet Neurol. 7, 1032-1043. doi: 10.1016/S1474-4422(08)70223-0

Fels, M., Bauer, R., and Gharabaghi, A. (2015). Predicting workload profiles of brain-robot interface and electromygraphic neurofeedback with cortical resting-state networks: personal trait or task-specific challenge? J. Neural Eng. 12:046029. doi: 10.1088/1741-2560/12/4/046029

Friedman, J. H. (1989). Regularized Discriminant Analysis. J. Am. Stat. Assoc. 84, 165-175. doi: 10.1080/01621459.1989.10478752

Gharabaghi, A. (2016). What turns assistive into restorative brain-machine interfaces? Front. Neurosci. 10:456. doi: 10.3389/fnins.2016.00456

Gharabaghi, A., Kraus, D., Leão, M. T., Spüler, M., Walter, A., Bogdan, M., et al. (2014a). Coupling brain-machine interfaces with cortical stimulation for brain-state dependent stimulation: enhancing motor cortex excitability for neurorehabilitation. Front. Hum. Neurosci. 8:122. doi: 10.3389/fnhum.2014.00122

Gharabaghi, A., Naros, G., Khademi, F., Jesser, J., Spüler, M., Walter, A., et al. (2014b). Learned self-regulation of the lesioned brain with epidural electrocorticography. Front. Behav. Neurosci. 8:429. doi: 10.3389/fnbeh.2014.00429

Gomez-Rodriguez, M., Peters, J., Hill, J., Schölkopf, B., Gharabaghi, A., and Grosse-Wentrup, M. (2011). Closing the sensorimotor loop: haptic feedback facilitates decoding of motor imagery. J. Neural Eng. 8:36005. doi: 10.1088/1741-2560/8/3/036005

Hammer, E. M., Halder, S., Blankertz, B., Sannelli, C., Dickhaus, T., Kleih, S., et al. (2012). Psychological predictors of SMR-BCI performance. Biol. Psychol. 89, 80-86. doi: 10.1016/j.biopsycho.2011.09.006

Heremans, E., Helsen, W. F., De Poel, H. J., Alaerts, K., Meyns, P., and Feys, P. (2009). Facilitation of motor imagery through movement-related cueing. Brain Res. 1278, 50-58. doi: 10.1016/j.brainres.2009.04.041
Heremans, E., Nieuwboer, A., Feys, P., Vercruysse, S., Vandenberghe, W., Sharma, N., et al. (2012). External cueing improves motor imagery quality in patients with Parkinson disease. Neurorehabil. Neural Repair 26, 27-35. doi: $10.1177 / 1545968311411055$

Hochberg, L. R., Bacher, D., Jarosiewicz, B., Masse, N. Y., Simeral, J. D., Vogel, J., et al. (2012). Reach and grasp by people with tetraplegia using a neurally controlled robotic arm. Nature 485, 372-375. doi: 10.1038/nature11076

Ivanova, G., Perez, D., and Both, R. (2005). Threshold adaptation for mean value based operant conditioning. Conf. Proc. Annu. Int. Conf. IEEE Eng. Med. Biol. Soc. 4, 3612-3615. doi: 10.1109/iembs.2005.1617263

Kaiser, V., Kreilinger, A., Müller-Putz, G. R., and Neuper, C. (2011). First steps toward a motor imagery based stroke BCI: new strategy to set up a classifier. Front. Neurosci. 5:86. doi: 10.3389/fnins.2011.00086

Kilavik, B. E., Zaepffel, M., Brovelli, A., MacKay, W. A., and Riehle, A. (2012). The ups and downs of beta oscillations in sensorimotor cortex. Exp. Neurol. 15-26. doi: 10.1016/j.expneurol.2012.09.014

Kraus, D., Naros, G., Bauer, R., Khademi, F., Leão, M. T., Ziemann, U., et al. (2016). Brain state-dependent transcranial magnetic closedloop stimulation controlled by sensorimotor desynchronization induces robust increase of corticospinal excitability. Brain Stimul. 9, 415-424. doi: 10.1016/j.brs.2016.02.007

Kraus, D., Naros, G., Bauer, R., Leão, M. T., Ziemann, U., and Gharabaghi, A. (2015). Brain-robot interface driven plasticity: distributed modulation of corticospinal excitability. Neuroimage 125, 522-532. doi: 10.1016/j.neuroimage.2015.09.074

Krucoff, M. O., Rahimpour, S., Slutzky, M. W., Edgerton, V. R., and Turner, D. A. (2016). Enhancing nervous system recovery through neurobiologics, neural interface training, and neurorehabilitation. Front. Neurosci. 10:584. doi: $10.3389 /$ fnins.2016.00584

Lee, T. D., Swanson, L. R., and Hall, A. L. (1991). What is repeated in a repetition? Effects of practice conditions on motor skill acquisition. Phys. Ther. 71, $150-156$.

Liepert, J., Greiner, J., Nedelko, V., and Dettmers, C. (2012). Reduced upper limb sensation impairs mental chronometry for motor imagery after stroke: clinical and electrophysiological findings. Neurorehabil. Neural Repair 26, 470-478. doi: $10.1177 / 1545968311425924$

Lotte, F., Larrue, F., and Mühl, C. (2013). Flaws in current human training protocols for spontaneous Brain-Computer Interfaces: lessons learned from instructional design. Front. Hum. Neurosci. 7:568. doi: 10.3389/fnhum.2013.00568

Mima, T., Matsuoka, T., and Hallett, M. (2001). Information flow from the sensorimotor cortex to muscle in humans. Clin. Neurophysiol. Off. J. Int. Fed. Clin. Neurophysiol. 112, 122-126. doi: 10.1016/S1388-2457(00) 00515-0

Morone, G., Pisotta, I., Pichiorri, F., Kleih, S., Paolucci, S., Molinari, M., et al. (2015). Proof of principle of a brain-computer interface approach to support poststroke arm rehabilitation in hospitalized patients: design, acceptability, and usability. Arch. Phys. Med. Rehabil. 96, S71-S78. doi: 10.1016/j.apmr.2014.05.026

Naros, G., and Gharabaghi, A. (2015). Reinforcement learning of self-regulated $\beta$-oscillations for motor restoration in chronic stroke. Front. Hum. Neurosci. 9:391. doi: 10.3389/fnhum.2015.00391

Naros, G., Naros, I., Grimm, F., Ziemann, U., and Gharabaghi, A. (2016). Reinforcement learning of self-regulated sensorimotor $\beta$ oscillations improves motor performance. NeuroImage 134, 142-152. doi: 10.1016/j.neuroimage.2016.03.016

Neuper, C., Wörtz, M., and Pfurtscheller, G. (2006). ERD/ERS patterns reflecting sensorimotor activation and deactivation. Prog. Brain Res. 159, 211-222. doi: 10.1016/S0079-6123(06)59014-4

Nijboer, F., Furdea, A., Gunst, I., Mellinger, J., McFarland, D. J., Birbaumer, N., et al. (2008). An auditory brain-computer interface (BCI). J. Neurosci. Methods 167, 43-50. doi: 10.1016/j.jneumeth.2007.02.009

Page, S. J., Dunning, K., Hermann, V., Leonard, A., and Levine, P. (2011). Longer versus shorter mental practice sessions for affected upper extremity movement after stroke: a randomized controlled trial. Clin. Rehabil. 25, 627-637. doi: 10.1177/0269215510395793

Parker, J., Mountain, G., and Hammerton, J. (2011). A review of the evidence underpinning the use of visual and auditory feedback for computer technology 
in post-stroke upper-limb rehabilitation. Disabil. Rehabil. Assist. Technol. 6, 1-8. doi: 10.3109/17483107.2011.556209

Pfurtscheller, G., Neuper, C., Brunner, C., and da Silva, F. L. (2005). Beta rebound after different types of motor imagery in man. Neurosci. Lett. 378, 156-159. doi: 10.1016/j.neulet.2004.12.034

Pichiorri, F., Morone, G., Petti, M., Toppi, J., Pisotta, I., Molinari, M., et al. (2015). Brain-computer interface boosts motor imagery practice during stroke recovery. Ann. Neurol. 77, 851-865. doi: 10.1002/ana.24390

Pimentel, M. A. F., Clifton, D. A., Clifton, L., and Tarassenko, L. (2014). A review of novelty detection. Signal Process. 99, 215-249. doi: 10.1016/j.sigpro.2013.12.026

Royter, V., and Gharabaghi, A. (2016). Brain state-dependent closed-loop modulation of paired associative stimulation controlled by sensorimotor desynchronization. Front Cell Neurosci. 10:115. doi: 10.3389/fncel.2016.00115

Rossiter, H. E., Boudrias, M.-H., and Ward, N. S. (2014). Do movement-related beta oscillations change following stroke? J. Neurophysiol. 112, 2053-2058. doi: $10.1152 /$ jn.00345.2014

Schnotz, W., and Kürschner, C. (2007). A reconsideration of cognitive load theory. Educ. Psychol. Rev. 19, 469-508. doi: 10.1007/s10648-007-9053-4

Shelton, F. N., and Reding, M. J. (2001). Effect of lesion location on upper limb motor recovery after stroke. Stroke 32, 107-112. doi: 10.1161/01.STR.32.1.107

Sherlin, L. H., Arns, M., Lubar, J., Heinrich, H., Kerson, C., Strehl, U., et al. (2011). Neurofeedback and basic learning theory: implications for research and practice. J. Neurother. 15, 292-304. doi: 10.1080/10874208.2011.623089

Sollfrank, T., Ramsay, A., Perdikis, S., Williamson, J., Murray-Smith, R., Leeb, R., et al. (2015). The effect of multimodal and enriched feedback on SMR-BCI performance. Clin. Neurophysiol. 127, 490-498. doi: 10.1016/j.clinph.2015.06.004

Stinear, C. M., Barber, P. A., Petoe, M., Anwar, S., and Byblow, W. D. (2012). The PREP algorithm predicts potential for upper limb recovery after stroke. Brain 135, 2527-2535. doi: 10.1093/brain/aws146

Sugiyama, M., Yamada, M., and du Plessis, M. C. (2013). Learning under nonstationarity: covariate shift and class-balance change: learning under nonstationarity. Wiley Interdiscip. Rev. Comput. Stat. 5, 465-477. doi: 10.1002/wics. 1275

Theodoridis, S., and Koutroumbas, K. (2009). Pattern Recognition. Burlington, MA; London: Academic Press.
Vidaurre, C., Kawanabe, M., von Bünau, P., Blankertz, B., and Müller, K. R. (2011a). Toward unsupervised adaptation of LDA for brain-computer interfaces. IEEE Trans. Biomed. Eng. 58, 587-597. doi: 10.1109/TBME.2010. 2093133

Vidaurre, C., Sannelli, C., Müller, K.-R., and Blankertz, B. (2011b). Coadaptive calibration to improve BCI efficiency. J. Neural Eng. 8:25009. doi: 10.1088/1741-2560/8/2/025009

Vukelić, M., Bauer, R., Naros, G., Naros, I., Braun, C., and Gharabaghi, A. (2014). Lateralized alpha-band cortical networks regulate volitional modulation of beta-band sensorimotor oscillations. NeuroImage 87, 147-153. doi: 10.1016/j.neuroimage.2013.10.003

Vukelić M., and Gharabaghi, A. (2015a). Oscillatory entrainment of the motor cortical network during motor imagery is modulated by the feedback modality. Neuroimage 1, 1-11. doi: 10.1016/j.neuroimage.2015.01.058

Vukelić, M., and Gharabaghi, A. (2015b). Self-regulation of circumscribed brain activity modulates spatially selective and frequency specific connectivity of distributed resting state networks. Front. Behav. Neurosci. 9:181. doi: 10.3389/fnbeh.2015.00181

Wood, G., Kober, S. E., Witte, M., and Neuper, C. (2014). On the need to better specify the concept of "control" in brain-computer-interfaces/neurofeedback research. Front. Syst. Neurosci. 8:171. doi: 10.3389/fnsys.2014. 00171

Yuan, K. H., and Bentler, P. M. (1998). Robust mean and covariance structure analysis. Br. J. Math. Stat. Psychol. 51 (Pt 1), 63-88. doi: 10.1111/j.2044-8317.1998.tb00667.x

Conflict of Interest Statement: The authors declare that the research was conducted in the absence of any commercial or financial relationships that could be construed as a potential conflict of interest.

Copyright $\odot 2017$ Bauer and Gharabaghi. This is an open-access article distributed under the terms of the Creative Commons Attribution License (CC BY). The use, distribution or reproduction in other forums is permitted, provided the original author(s) or licensor are credited and that the original publication in this journal is cited, in accordance with accepted academic practice. No use, distribution or reproduction is permitted which does not comply with these terms. 